\title{
Tip-Enhanced Raman Analysis of Plasmonic and Photocatalytic Properties of Copper Nanomaterials
}

\author{
Zhandong $L i^{1}$, and Dmitry Kurouski*1,2
}

${ }^{1}$ Department of Biochemistry and Biophysics, Texas A\&M University, College Station, Texas

77843, United States

${ }^{2}$ Department of Biomedical Engineering, Texas A\&M University, College Station, Texas, 77843,

United States

\section{Contents}

\section{Experimental Details.}

Chemicals

Preparation of CuNWs and CuNCs

Modification of the molecular reporter monolayer on the $\mathrm{Cu}$ nanostructures

TERS Probe Fabrication

TERS measurement

\section{AFM images of CuNWs and CuNCs.}




\section{Experimental Details.}

\section{Chemicals.}

Copper $(\|)$ chloride dihydrate $\left(\mathrm{CuCl}_{2} \cdot 2 \mathrm{H}_{2} \mathrm{O}, 99+\%\right)$, hexadecylamine (HDA), glucose, iodine $\left(\mathrm{I}_{2}, 99.8+\%\right)$, 4-Mercaptophenyl)-methanol (MPM), 4-Mercaptobenzoic Acid (MBA), sodium hydroxide (NaOH, 98\%), 4-nitrobenzenethiol were purchased from Sigma-Aldrich (St. Louis, MO). Ethanol was purchased from Decon Labs (King of Prussia, PA). All chemicals were used as received without purification.

\section{Preparation of CuNWs and CuNCs.}

For CuNWs, $6.3 \mathrm{mg} \mathrm{mL}^{-1}$ copper $(\|)$ chloride dihydrate $\left(\mathrm{CuCl}_{2} \cdot 2 \mathrm{H}_{2} \mathrm{O}, 99+\%\right), 73.2 \mathrm{mg} \mathrm{mL}^{-1} \mathrm{HDA}(98 \%)$, and $15 \mathrm{mg} \mathrm{mL}^{-1}$ glucose $(99.5+\%)$ were dissolved in $3 \mathrm{~mL}$ of DI water. The solution was mixed for $10 \mathrm{~min}$ at $60{ }^{\circ} \mathrm{C}$ water bath and brought pinto a Teflon-lined autoclave. Then, the Teflon-lined autoclave was kept in $100^{\circ} \mathrm{C}$ oil bath and stirring for over $6 \mathrm{~h}$. The color of the mixture solution changed from light blue to dark red after the reaction finished. For the CuNWs and CuNCs mixtures, $0.1 \mathrm{mg}$ of iodine $\left(\mathrm{I}_{2}, 99.8+\%\right)$ were added in the above-mentioned mixture solution for CuNWs and kept in the same type Teflon-lined autoclave at $100{ }^{\circ} \mathrm{C}$ and stirred for overnight. The reactant solutions were further purified with DI water for several times with centrifuging at $5000 \mathrm{rpm}$.

Modification of the molecular reporter monolayer on the $\mathrm{Cu}$ nanostructures.

A drop of the as-synthesized CuNWs/CuNCs stock solution was first deposited on precleaned Si wafer, incubated for 0.5 h. Subsequently, the nanoplates deposited Si wafer was immersed in a $2 \mathrm{mM}$ ethanolic 4-NBT(or other molecular reporters) solution for $1 \mathrm{~h}$ to form monolayer on these $\mathrm{Cu}$ nanostructures. Finally, the modified sample was sonicated in ethanol for 3 min for removing the uncoordinated molecules.

TERS Probe Fabrication. Silicon AFM probes with related parameters force constant $2.7 \mathrm{~N} / \mathrm{m}$ and resonance frequency 50-80 kHz were purchased from Appnano (Mountain View, CA). Then, the metal evaporation was carried out for coating the AFM tips with a layer of gold. Briefly, two of the probes were fixed onto each of the clamped device and ten of the devices were put in the thermal evaporator chamber (MBrown, Stratham, NH). During metal deposition, the pressure was kept at $\sim 1 \times 10^{-6}$ mbar. Then, gold pellets (Kurt J. Lesker, Efferson Hills, PA) thermally evaporate at constant $0.2 \mathrm{~A} \cdot \mathrm{s}^{-1}$ rate. After $70 \mathrm{~nm}$ of Au was deposited on the AFM tips, the evaporation was stopped and kept cooling down to room temperature. The temperature at the tip surface and deposition chamber was $\sim 50^{\circ} \mathrm{C}$.

TERS measurement. AFM-TERS and AFM scanning was carried out on the AIST-NT-HORIBA system equipped with a $632.8 \mathrm{~nm}$ continuous wavelength $(\mathrm{CW})$ laser. Laser light was brought to the sample surface in a side-illumination geometry with a 100X Mitutoyo microscope objective. The scatterring electromagnetic radiation was also collected with 
the same objective and directed introduced to a fiber-coupled Horiba iHR550 spectrograph that equipped with a Synapse EM-CCD camera (Horiba, Edison, NJ). 

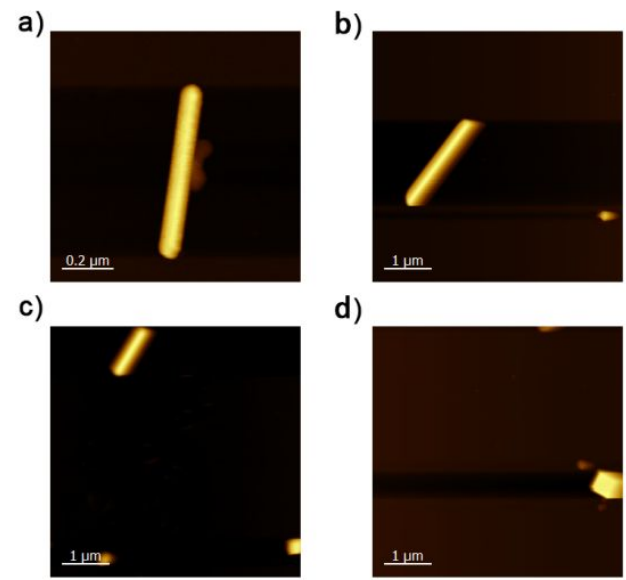

d)

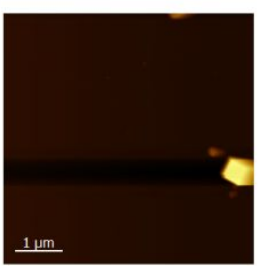

Figure S1. AFM images of a,b CuNWs. c,d CuNWs and CuNCs. 